\title{
Un debate actual sobre la formación inicial de docentes en México
}

\author{
Ruth Mercado Maldonado
}

\begin{abstract}
Resumen
El ensayo plantea un debate inscrito en el contexto de las discusiones sobre una reforma de carácter oficial y nacional anunciada en México, para la formación inicial de docentes. Dos posiciones son punto de partida; la primera consiste en concebir a la formación de docentes en tanto proceso continuo, lo cual implica actuar desde una visión integral y sistémica sobre los programas de formación docente. La segunda posición cuestiona las grandes y cíclicas reformas con las que se pretenden cambios radicales en subsistemas educativos nacionales y cuyas indeseables consecuencias se describen en los estudios del campo. La discusión central del trabajo remite al papel de las prácticas escolares en la formación inicial de docentes y está apoyada en literatura internacional sobre ese tema. El trabajo destaca la construcción de conocimiento sobre la escuela y la docencia que desarrollan los sujetos involucrados en las prácticas: profesores de escuelas, docentes de instituciones formadoras y estudiantes. Se plantea para el análisis la noción de "encuentro de dos culturas" la de escuelas de práctica y de las instituciones formadoras, señalando tanto dificultades como beneficios reconocidos por estudiantes y profesores.
\end{abstract}

Palabras clave: Formación de profesores, educación, enseñanza.

\section{A current debate on an initial education of teachers in Mexico}

\begin{abstract}
In this paper we discuss on the national reform for the education of new teachers in Mexico. We start from two positions: the first is to develop a teacher training as a continuous process which involves acting from a systemic and integral view on teacher education programs. The second position questions major and cyclical reforms which propose radical changes in national educational subsystems and whose undesirable consequences are described in studies in the area. The central I discussion refers to the role of school practice in initial teacher training and is supported by international literature on the subject. The work highlights the construction of knowledge about school and teaching which develop the subjects involved in the practices: school teachers, training institutions, teachers and students. We propose to analyze the notion of "meeting of two cultures," that is the meeting between the practice of schools and training institutions, bringing up both challenges and benefits recognized by students and teachers.
\end{abstract}

Keywords: Teacher education, education, teaching.

\section{Um debate atual sobre a formação inicial de docentes no México}

\section{Resumo}

O ensaio propõe um debate inscrito no contexto das discussões sobre uma reforma de caráter oficial e nacional anunciada no México para a formação inicial de docentes. Duas posições são ponto de partida: a primeira consiste em conceber a formação de docentes como um processo contínuo, o qual implica atuar a partir de uma visão integral e sistêmica dos programas de formação docente; a segunda posição questiona as grandes e cíclicas reformas com as quais se pretendem mudanças radicais em subsistemas educativos nacionais e cujas consequências indesejáveis descrevem-se nos estudos de campo. A discussão central do trabalho remete ao papel das práticas escolares na formação inicial de docentes e está apoiada em literatura internacional sobre esse tema. $\mathrm{O}$ trabalho destaca a construção de conhecimento sobre a escola e a docência que os sujeitos envolvidos nas práticas desenvolvem: professores de escolas, docentes de instituições formadoras e estudantes. Propõese, para a análise, a noção de "encontro de duas culturas", a da prática desenvolvida na escola e a das instituições formadoras, assinalando tanto as dificuldades como os benefícios reconhecidos por estudantes e professores.

Palavras-chave: Formação de professores, educação, ensino. 
De entre todas las actividades que son profesionales o aspiran a serlo, la enseñanza es la única a la que se ha encargado la tarea formidable de crear capacidades y destrezas que permitirán a las sociedades sobrevivir y tener éxito en la era de la información.

Hargreaves

\section{Introdução}

Organizo el presente texto en tres partes, en la primera planteo en qué términos me referiré a la formación docente; en segundo lugar identifico algunas de las prioridades a revisar en este campo, en México y algunos países de la región. Por último propongo considerar estas prioridades frente a los retos que aún están pendientes en la formación de docentes y la educación básica.

Actualmente, podríamos coincidir en que, como proclaman las más diversas voces, no es posible aspirar a mejorar la calidad y equidad educativas, sin propiciar una mejor formación, tanto de los futuros docentes como de quienes están en servicio. Sin embargo este entendimiento lleva también, en ocasiones, a responsabilizar al profesorado de los "malos resultados" escolares que en nuestras latitudes continúan reportándose. Ya diversos estudiosos del tema han llamado la atención acerca de que no sólo se requieren mejores docentes, sino cambios profundos en las dimensiones organizativas y de gestión de los sistemas escolares donde prestan sus servicios.

Por otro lado, debemos reconocer que es necesario el debate, el análisis y la revisión de propuestas orientadas a mejorar la formación docente en nuestro país y a ese respecto comenzaré por ubicar en qué términos me referiré a ésta.

La literatura internacional acerca de cómo entender la formación de los maestros es muy vasta e incluye perspectivas diversas; sin embargo, puede decirse que, en general, hay ciertas coincidencias sobre algunos de los aspectos que deberían atenderse prioritariamente en este tema.

Una de ellas es la necesidad de arribar a concepciones y políticas integrales y sistémicas al tratarse de la formación de maestros. En ese sentido, una de las tendencias que ha tomado fuerza en este campo, es la de concebir a la formación de docentes como un proceso continuo.

Desde esa visión, el proceso formativo comienza aún antes de la formación inicial, prosigue en ésta y posteriormente, al ejercer la práctica profesional (Vaillant, 2002). Esta última, en términos de Schön, se trataría del denominado "continum de la práctica" (1992), en el cual los sujetos desarrollan conocimientos necesarios para el ejercicio profesional.

Ávalos $(2000,2004)$ revisa cómo, para la formación docente chilena, esta idea, de la formación como proceso continuo, estuvo presente desde la sexta década del siglo $X X$, aunque dice, es a partir de los noventa que "empiezan a formularse políticas integrales de formación docente que comprenden ambas etapas, la de formación inicial y la que tiene lugar durante el ejercicio profesional." (2000, pp. 18-
19). Igualmente, en los Estándares Chilenos de Desempeño para la Formación Inicial de Docentes se propone que: "Ia formación inicial permitirá al futuro educador o educadora [“. ..."] enfrentar las primeras experiencias de enseñanza y [“...."] capacitarlo para emprender [“...."] la segunda etapa de su formación que tendrá lugar durante toda su trayectoria profesional" (MEC, 2000).

En el caso mexicano, ya se planteaba en un documento para la discusión nacional, posterior a la reforma de los noventa a la educación normal', que: "La formación docente se concibe como un proceso de aprendizaje permanente, ya que las competencias y conocimientos que adquiere un maestro son resultado, no sólo de la formación inicial, sino de los aprendizajes que realiza durante el ejercicio de su profesión..." (Secretaría de Educación Pública, 2003, p. 12). ${ }^{2}$

Así mismo, en los actuales planes de estudios para la formación inicial en México, se reconoce a ésta como un momento de la formación docente. Se dice en uno de esos planes, que no todas las necesidades de formación se pueden cubrir "de manera específica y con certeza, mediante la formación inicial, pero constituyen retos estimulantes para continuar la preparación docente, es decir, para asumir la formación permanente durante el servicio" (Secretaría de Educación Pública, 2004, p. 54).

De acuerdo con esta concepción de la formación docente, en diferentes partes del mundo en la última década, se han promovido políticas tendientes al desarrollo de verdaderos Sistemas de Formación Continua. En éstos, las tendencias más avanzadas proponen articular las perspectivas de formación inicial con las de la formación en servicio, sin perder de vista la especificidad de cada una.

Es en este marco de la formación docente entendida como un proceso continuo que ubico la presente exposición.

\section{Una prioridad en el campo de la formación docente: su necesaria relación con la práctica escolar.}

Es conocida la perspectiva que en la formación de los profesionales propone la importancia que para ésta tiene la proximidad con la práctica. En el caso de los futuros docentes, se trata de la cercanía con la práctica profesional que tiene lugar en las escuelas. Para los profesores en servicio, esta postura implica que los programas dirigidos a este sector, consideren la experiencia y conocimiento que ellos desarrollan en el ejercicio cotidiano de la docencia, en lugar de suponer de antemano su incompetencia profesional (Encinas, 2005; Souza, 2007).

Por ahora sólo me remitiré a la formación inicial que, en mi perspectiva, tiene un papel importante en la historia

1 Aquella en que se hacen los estudios y la práctica necesarios para obtener el título de maestro de primera enseñanza (Nota de revisión).

2 En México, la formación inicial de los docentes se realiza en las escuelas normales que tienen nivel de licenciatura, es decir, de educación superior equivalente a las carreras universitarias y con los mismos años de estudio. 
profesional de los docentes, como un trayecto donde se introducen al conocimiento sistematizado de disciplinas asociadas con la educación y, parcialmente, a la práctica escolar y a la de enseñanza.

Sobre las prácticas escolares en la formación inicial, desde antes de las reformas realizadas en países de la región en los años noventa, se ha propuesto acercar de una manera sostenida a los futuros profesores a las culturas escolares donde ejercerán su profesión. Es decir, a la vida escolar tal como ésta sucede y al trabajo de los maestros en servicio para conocer y aprender de esa realidad.

Así, en diferentes partes del mundo, no sólo en América Latina, los planes de estudio para la formación inicial y los distintos programas articulados a esos planes, han otorgado gran importancia al acercamiento de los futuros docentes hacia las escuelas donde posteriormente ejercerán como profesionales. Un supuesto de base que comparten estas propuestas, aunque con diferentes matices y perspectivas teóricas, es el de considerar que el aprendizaje de la docencia tiene lugar de manera importante (aunque no exclusivamente) en la práctica, en contacto con las condiciones reales de la vida escolar y sus pautas culturales.

Algunos autores lo señalan de esta manera:

En el recorte temporal de los programas (de formación inicial) debe acordarse un lugar sustancial a la formación práctica en el medio escolar, prácticas de larga duración, contactos repetidos y frecuentes con los medios de práctica, clases centradas en el análisis de las prácticas, análisis de casos, solución de problemas concretos, etcétera. (Tardif, Figueroa, Cividini, \& Mujawamariya, 2000, p. 51).

Coincidiendo con esa posición, encuentro en mi propio trabajo que existen grandes posibilidades formativas al desarrollarse en esos términos la formación de los futuros docentes; comenzamos ya a identificarlas y documentarlas en estudios de posgrado sobre el tema (Estrada, 2009; Estrada \& Mercado, 2008; Hilario 2010) ${ }^{3}$. Sin embargo, hace falta más investigación para conocer cuáles son los procesos formativos que realmente tienen lugar durante las prácticas escolares de los estudiantes de magisterio, más allá de las posiciones que niegan o afirman las ventajas que pueden tener estas experiencias de práctica para su formación.

En nuestro país, con el plan de estudios vigente, se ha propiciado el acercamiento paulatino de los futuros maestros a la realidad escolar y las prácticas de enseñanza en las condiciones reales en las que ésta ocurre durante el último año de su licenciatura. ${ }^{4}$ La orientación de ese acercamiento

3 Deben también reconocerse y publicarse los estudios que en las propias instituciones formadoras se hacen sobre estos procesos y que aportan conocimiento que puede servir de base para mejorar las prácticas escolares en la formación inicial.

4 No existirían mejores "situaciones auténticas de aprendizaje" cuyo diseño se propone en el nuevo modelo curricular para la formación docente inicial que se elabora desde la Secretaría de Educación Pública (SEP) y al que me referiré más adelante. se dirige a quien está aprendiendo a conocer los espacios educativos escolares, así como a las herramientas culturales y a profesionales existentes en ellos. En ese proceso están apoyados por las materias de contenido teórico presentes también en el currículum de la formación inicial. Se trata con ello de colocar a los estudiantes en situación de aprendizaje, articulando las experiencias de la práctica con la teoría respecto a la realidad escolar y a la enseñanza que en ésta tiene lugar.

En ese sentido, los planes de estudio para la formación inicial en sus diferentes variantes en México, reconocen explícitamente el carácter formativo de la experiencia de los estudiantes en las escuelas de educación básica, como se dice en uno de ellos: "El futuro maestro [de educación especial] se formará en las aulas de la escuela normal, en los servicios de educación especial y en las escuelas regulares de educación básica" (SEP, 2004, p. 56).

Y continúa el mismo Plan:

La observación y la práctica [de los estudiantes] no se realizan con el fin de calificar o criticar lo que sucede en el aula, ni de identificar un modelo de docencia que se deba imitar; sino de registrar información para analizar y explicar las formas de proceder de los maestros [“...."] y para reconocer prácticas escolares adecuadas a las características de los grupos y de cada uno de los alumnos (SEP, 2004, p. 58).

Así mismo, en todos los planes de estudio para la formación inicial, se reconoce la importancia formativa que tiene para los estudiantes trabajar en los grupos de la escuela básica al lado de un profesor "experimentado" de ese nivel, llamado "tutor", como lo señala uno de ellos:

Se espera que los profesores [de escuelas básicas], cumplan una función de tutoría durante las observaciones y las prácticas educativas guiando a los estudiantes en los procedimientos y en la toma de decisiones adecuadas [“...."] y transmitiendo sus saberes y experiencia... (SEP, 2004, p. 59).

En otros países de la región el planeamiento es semejante. Un reporte de Chile nos habla de que en este país, con la reforma de 1997, las actividades referidas a las prácticas escolares de los futuros docentes en el currículum "aumentaron desde aproximadamente un $8 \%$ en los años ochenta hasta un promedio cercano al 20\%" (Ávalos, 2004, p. 13).

Adoptar esa perspectiva en la formación inicial es acorde, en términos generales, con lo que se plantea en actuales investigaciones sobre el papel de la práctica en la formación de los profesionales, en particular de los docentes. Además de autores que se han ocupado específicamente de este tema (Ávalos, 2004; Espinosa, 2007; Mercado, 2002; Rockwell \& Mercado, 1986; Schön, 1992; Tardif e cols., 2000; Tardif, Lessard, \& Lahaye, 1991), existen desarrollos teóricos sobre el aprendizaje situado que permiten sustentar las propuestas formativas apoyadas en la práctica escolar.

Por ejemplo, Lave y Wenger (1991) conciben el desarrollo del aprendizaje profesional asociado al proceso denomi- 
nado "participación periférica legítima en las comunidades de práctica", en el cual tienen lugar constantes negociaciones de significado entre los participantes de dichas comunidades. Así mismo, la formación profesional se concibe como un proceso inconcluso, como una carrera de vida en la que siempre se encuentra presente la construcción de nuevos aprendizajes.

Esos aprendizajes se producen en el intento por resolver los problemas reales que se presentan en la práctica (Lave, 1988; Lave \& Wenger, 1991; McLellan, 1991; Schubaver-Leoni \& Grossen, 1992;) y que Schön llama conocimiento en la acción (1992), Clandinin y Connelly (1996) conocimiento profesional y repertorios de la práctica, en el caso de Wenger (1998). ${ }^{5}$

Wenger (1998) señala, igualmente, que el desarrollo de competencias profesionales y destrezas para la solución de problemas de la práctica profesional tienen lugar en el proceso de la participación en las comunidades de práctica. La participación periférica legítima es para Lave y Wenger (1991) una manera de pertenecer a una comunidad de práctica.

La participación, señalan dichos autores, deja de ser periférica, cuando los profesionistas se integran a una comunidad de práctica y se van convirtiendo, paulatinamente, en participantes centrales de la práctica profesional. La participación en las comunidades de práctica se puede dar de manera periférica mediante distintos niveles de aproximación; en nuestro caso, como sucede con las prácticas escolares de los estudiantes de magisterio.

Por medio de las prácticas se tiene acceso a un espacio que no se encuentra completamente dentro, ni totalmente fuera de la comunidad de práctica (Wenger, 1998). De esa manera "Los maestros aprenden en las comunidades de práctica, en la participación periférica legítima..." (Lave \& Wenger, 1991).

El repertorio de una comunidad de práctica, dice Wenger, incluye rutinas, palabras, instrumentos, maneras de hacer, relatos, gestos, símbolos, géneros, acciones o conceptos que la comunidad ha producido o adoptado en el curso de su existencia y que han pasado a formar parte de su práctica. Incluye el discurso por el que los miembros de la comunidad crean afirmaciones significativas sobre el mundo, además de los estilos por medio de los cuales expresan sus formas de afiliación y su identidad como miembros (Wenger, 1998).

Habría tres posiciones en las que coinciden los autores antes señalados: a) el aprendizaje tiene un carácter social y personal; b) el aprendizaje es construido; c) la construcción del aprendizaje tiene lugar en situaciones determinadas de la práctica social; es decir, que el aprendizaje se desarrolla durante los procesos de participación en las comunidades de práctica. Podríamos agregar, como en las escuelas, si las pensamos en términos de comunidades de práctica. ${ }^{6}$

5 Un desarrollo más amplio sobre estas aportaciones teóricas referidas, en su caso, a la apropiación de nuevas propuestas pedagógicas por los docentes puede verse en Espinosa (2007).

6 Estas ideas están desarrolladas con mayor amplitud en el estudio de Estrada (2009) sobre las prácticas de pre-profesionales en la formación inicial.
Los anteriores planteamientos teóricos relativos al carácter formativo de la práctica, que son tributarios, a su vez, de las antecedentes aportaciones de Vigotsky sobre aprendizaje, ${ }^{7}$ no niegan en forma alguna la necesidad de la formación teórica de los futuros profesionales. Plantean en cambio, la imposibilidad de formarlos sin considerar de manera preponderante el papel de la práctica en ese proceso. Así lo explican claramente los actuales planes de estudio para la formación inicial en el país. ${ }^{8}$

La actual formación inicial en México, próxima a los anteriores planteamientos teóricos en cuanto a las prácticas escolares, aún no desarrolla todo su potencial, el cual hemos comenzado a identificar. Sabemos ya que las prácticas escolares vinculadas a las demás líneas curriculares de los planes de estudio en las normales, están generando procesos formativos importantes que es necesario reconocer, así como aquellos aspectos que deben mejorarse. Ambas cuestiones deben considerarse antes de tomar decisiones sobre cambios curriculares a la formación inicial.

\section{Las prácticas escolares de los futuros docentes: encuentro entre dos culturas, un añejo y a la vez actual reto en la formación inicial.}

Uno de los aspectos más sensibles que hemos identificado en la formación inicial mexicana, con los actuales planes de estudio, se ubica en el espacio donde interactúan las escuelas de formación y las de educación básica. Podemos llamarlo el espacio del encuentro entre dos culturas, que durante su historia han estado siempre en contacto, pero no de la manera actual. Le llamaría así, implicando que entre ambas culturas existe un espacio de construcción de procesos entre los sujetos participantes, más que una "laguna" entre esos dos mundos como se le llama en otros estudios (Marcelo, 1999, p. 6).

En el caso de México, este encuentro intercultural, tiene lugar a lo largo de la licenciatura, principalmente durante las prácticas de pre-profesionales de los estudiantes, en los dos últimos semestres de los ocho que conforman a la formación inicial.

Al interactuar estas instituciones, ahora ambas concebidas como espacios de formación, se desarrollan complejos procesos de negociación de significados (Wenger, 1998) entre los sujetos involucrados, de aportaciones e intercambios mutuos, encuentros y desencuentros, entre otros. Estos procesos ocurren en las relaciones que tienen lugar durante las prácticas entre los asesores y estudiantes de las instituciones formadoras por un lado, y por otro, los tutores o maestros de las escuelas de práctica; estas tres figuras constituyen lo

7 Para Vigotsky el elemento fundamental de los cambios de los sujetos radica en la relación que éstos establecen con la sociedad y la cultura (Cole \& Scribner, 1978).

8 No por ello podrían calificarse de "empiristas" como lo hacen algunos especialistas que proponen ahora su derogación. Además de tratarse de un término peyorativo, desde él se ignora que la ciencia misma trabaja con lo empírico, tanto como con la teoría. 
que algunos autores denominan la tríada de las prácticas de formación inicial (Wilson, 2006). ${ }^{9}$

Una de las cuestiones que hemos documentado al estudiar las relaciones entre estas tres figuras es, por ejemplo cómo, desde la institución formadora asesores y estudiantes tratan con dificultades de ajustarse a los ritmos y las necesidades propias del trabajo en las escuelas de práctica. En algunos casos, desde el espacio de la formación se percibe que las formas de enseñanza de los profesores tutores son diferentes a las que llevan los estudiantes para practicar.

Suele también aspirarse, desde la institución formadora, a que los tutores adopten los modelos de enseñanza que aquella diseña con sus estudiantes, o que les permitan practicar de acuerdo con esas propuestas. También se escucha en los espacios de formación que es difícil para los estudiantes aprender a ser maestros con tutores que, por ejemplo "aprovechan" su presencia para "encargarles el grupo" mientras atienden otras tareas.

Aquello que los formadores desearían para las prácticas de sus estudiantes, proviene de lo que les demanda su plan de estudios, así como de sus propias percepciones sobre cómo deberían ser la escuela básica y la docencia.

En diversas latitudes se presentan percepciones semejantes hacia las escuelas de práctica, desde las instituciones formadoras, como se dice en la siguiente referencia: "Los centros de formación inicial de docentes, han desarrollado tradiciones, formas de trabajo y adoptan posiciones sobre cómo deben ser la formación y la docencia, que son diferentes a las de los maestros de escuelas de práctica" (Tardif e cols., 2000, pp. 54-57).

En general, en los centros de formación inicial se asume que es ahí donde se enseña a ser maestro y que en las escuelas sólo se practica lo aprendido, creencia que proviene de anteriores perspectivas sobre la formación inicial y la práctica docente cotidiana.

Los profesores de las escuelas de práctica, a su vez, se encuentran inmersos en tareas diferentes a las de las instituciones formadoras. Ellos trabajan ante prioridades y necesidades distintas a las que enfrentan los maestros y estudiantes de la formación inicial; tienen plazos para llegar a determinadas metas con sus alumnos. Deben llegar al fin de cursos tratando de alcanzar las metas esperadas con sus alumnos, más que guiar a los estudiantes de magisterio. Saben que en última instancia los únicos responsables de los resultados finales de sus alumnos son ellos mismos, ante las autoridades y los padres de familia.

En todo caso, la tutoría a los futuros docentes no es prioritaria en el trabajo de los profesores de escuelas básicas, ni tendría por qué serlo, sólo pueden orientarlos como otra de sus tareas. Además, tienen sus propias ideas sobre cómo se

9 Destaco a estos tres sujetos como los que intervienen en el diseño y decisiones que orientan las prácticas en lo pedagógico, aunque en las prácticas escolares para la formación inicial hay otros importantes participantes, como los alumnos de las escuelas básicas, los padres de familia, los directores de escuela, entre otros. enseña y cómo se aprende a enseñar, las cuales pueden diferir de las que proponen los maestros de formación inicial.

Por otro lado, aunque los tutores acepten a un estudiante de magisterio en su grupo, y muchos lo hagan con entusiasmo, no siempre existe claridad sobre los límites de esa función. Los tutores perciben que es un trabajo agregado a las innumerables actividades que ya deben cumplir cotidianamente.

Los estudiantes, por su parte no parecen creer que es posible aprender de lo que ocurre en los grupos donde practican y de cómo sus tutores ejercen la docencia. Suelen decir que no pueden llevar a cabo la enseñanza como se les propone en las normales, porque los tutores no aprecian que así mejore el aprovechamiento de los alumnos.

Sin embargo, asesores de normal, tutores de escuelas básicas y estudiantes de magisterio, han desarrollado durante más de diez años las prácticas escolares, participando así en procesos formativos cuyos contenidos es necesario reconocer.

Los asesores de prácticas en las normales por ejemplo, han construido lo que podríamos llamar una verdadera especialización dentro de las funciones académicas de la normal. Han aprendido, en muchos casos, cómo llegar a acuerdos con los profesores tutores sobre la conducción de los estudiantes.

Igualmente, como asesores han aprendido a conocer las distintas posibilidades de los estudiantes frente a los problemas que enfrentan al practicar la enseñanza bajo condiciones reales, así como a encontrar los mejores apoyos para cada caso. También han aprendido que la vida y el trabajo en las escuelas de práctica imponen condiciones a veces no imaginadas a las prácticas de los estudiantes ante las cuales ellos deben conducirlos.

Por lo que toca a los tutores, muchas veces se han involucrado en las prácticas de los futuros docentes de manera cercana, apoyándolos en sus dificultades ante los grupos. Encuentran entre los repertorios de su experiencia, recursos qué compartir con los estudiantes en términos de que se inicien en la acción docente; por ejemplo, para ser capaces de construir con los niños acuerdos de convivencia y trabajo en el aula (Estrada, 2009). O bien identificando el valor que puede tener para su propia formación lo que llevan los estudiantes, como el acercamiento a las tecnologías informáticas, para ellos un tanto ajenas hasta entonces (Hilario, 2010).

Para los estudiantes, las prácticas escolares también representan situaciones de aprendizaje, pues reportan experimentar procesos de cambio en sus expectativas sobre el trabajo docente. Describen, cómo a lo largo de ese período en las escuelas, muchas veces transforman sus ideas iniciales acerca de la enseñanza y la vida escolar, o de los sujetos que en ella participan, así como de los procesos que tienen lugar entre ellos. En esas descripciones logran identificar, en ocasiones, aquello que han aprendido y que es diferente a lo que conocían desde la normal.

Los estudiantes reconocen haberse orientado inicialmente en sus prácticas, por expectativas negativas hacia la escuela y sus tutores, así como haber anticipado una simpli- 
cidad en la enseñanza muy alejada de su real complejidad, que posteriormente empezaron a comprender (Estrada, 2009).

Sin embargo, las experiencias y procesos formativos sobre la docencia donde participan las tres figuras de la tríada de las prácticas, son aún poco reconocidos ${ }^{10}$ y aprovechados en los espacios que deberían dedicarse a ello en la formación de los estudiantes.

Por ejemplo, el trabajo en las normales sobre las experiencias formativas de los estudiantes en las escuelas de práctica tendría que aproximarlos a la investigación, ya muy vasta actualmente, que da cuenta de los procesos que constituyen a las escuelas y a la enseñanza, inscritas en contextos sociales particulares donde ellos ejercerán su profesión y no sólo a los modelos de lo que ellas deben ser.

Se trataría de que los futuros docentes conocieran los procesos de construcción de conocimiento, de reproducción, pero también de creación y aún de innovación que tienen lugar en esas escuelas y que puedan reconocer las situaciones que dentro de ese ámbito, en algunos casos constriñen, pero en otros posibilitan el ejercicio docente significativo. ${ }^{11}$

Así lo señalan los autores de un trabajo sobre este tema que nos dicen:

En los Estados Unidos y Canadá se cuentan actualmente varios millares de investigaciones efectuadas en las [aulas de escuelas básicas]. Este movimiento (de investigación) se basa en el reconocimiento de que los maestros de oficio son poseedores de saberes (conocimientos, competencias, actitudes) que la investigación debe esforzarse en actualizar y en integrar a los programas de formación inicial. (Tardif e cols., 2000, p. 53).

No obstante, los asesores, poco se detienen en los seminarios donde podrían apoyar más fuertemente las prácticas de los estudiantes, pues actualmente deben priorizar la elaboración del documento recepcional, dados los lineamientos a ese respecto del plan de estudios vigente. Con ello, se provoca que los asesores, vean limitadas sus posibilidades de dedicarse con sus estudiantes a desarrollar análisis más formativos sobre los problemas pedagógicos con los que éstos se encuentran durante sus prácticas.

La negociación entre estas dos culturas a que me he referido, se ha dado con matices muy diferentes en cada lugar, con mayores frutos y procesos favorables en algunos casos y desfavorables o frustrantes en otros.

De cualquier manera, el comienzo de un acercamiento más provechoso desde la formación inicial que tome lo mejor de las prácticas docentes cotidianas ha tenido lugar, no

10 Marcelo (1999) plantea, en cambio, que la historia entre las escuelas primarias y las de formación docente en las prácticas de enseñanza, se caracterizan más por los desencuentros y la ignorancia recíproca, que por una colaboración entre ambas.

11 También deben actualizarse las asignaturas teóricas del currículum de formación inicial con las aportaciones científicas más recientes, así como las didácticas especializadas. sabemos con qué resultados hasta ahora. Haría falta conocer más sobre lo que ha ocurrido con las prácticas escolares en la formación inicial durante más de una década y con las cuales se pretendió acortar el abismo entre esa formación y la realidad escolar.

En cambio, cuando se habla de los cambios necesarios para mejorar la formación inicial en nuestro país, lo que suele proponerse en los últimos años, es el traslado de esa formación a los espacios universitarios. A ese respecto, debíamos saber o recordar que esa medida, no ha implicado por sí misma, mejorar las áreas más críticas de la formación inicial en los países que así lo han hecho, por ejemplo, Chile. La relación entre la formación teórica y práctica, así como las posibilidades de encuentro productivo entre la institución formadora y las escuelas de educación básica, no son problemas resueltos en los países que han hecho ese traslado, ni en aquellos donde la formación docente se ha hecho prioritariamente en las universidades. ${ }^{12}$

Pese a lo anteriormente expuesto, en el sentido de que aún tenemos un camino por recorrer, para contar con más y mejor conocimiento sobre los procesos formativos que se han construido en las normales para los futuros docentes en nuestro país, parece que estamos frente a una nueva reforma, por cierto, no anunciada oficialmente. Es por ello que hablando de prioridades en la formación de docentes plantearía una más, sólo que a manera de pregunta:

\section{La reforma a la formación inicial ¿es ahora una prioridad?}

¿Por qué ahora una reforma a la formación inicial? Habría, como en toda reforma, innumerables razones que podrían argumentarse por quienes la promueven. No obstante, sabemos que un Estado no emprende la reforma curricular de un subsistema de la importancia de la educación normal en México, sólo por motivos académicos, sino que los tiempos políticos influyen de manera importante en este tipo de decisiones.

Por otro lado, se ha planteado entre los documentos ${ }^{13}$ que circulan de manera informal acerca de esa reforma, que una de las razones por las que ésta se justifica es la de vincular la formación docente inicial con la reforma a la educación básica actualmente en proceso.

Sin embargo, esta reforma a la educación básica, está efectivamente aún en proceso y algunos de sus componen-

12 Se aduce también que así se terminaría con la "endogamia" de las normales, aunque se desconoce que, por ejemplo, desde la reforma de 1984, el personal docente universitario en esas instituciones haya aumentado paulatinamente. Además, habría otras formas de promover una mayor cercanía entre las normales y el sistema de educación superior universitario.

13 "Modelo Integral para la Formación Profesional y el Desarrollo de Competencias del Maestro de Educación Básica." Y "Modelo Curricular para la Formación Profesional de los Maestros de Educación Básica." Ambos del 2010 y marcados como de "Circulación interna" de la Secretaría de Educación Pública. 
tes podrían hacerse del conocimiento de los estudiantes de magisterio sin cambiar los planes de estudio vigentes.

Además, la reforma a la educación básica está por mostrar sus posibilidades de consolidarse como una reforma con la calidad y consistencia pedagógica suficientes, lo que no se ha mostrado en su proceso de elaboración y de implementación. Este proceso ha implicado un debate nacional aún abierto sobre la pertinencia de esa reforma en la perspectiva en que se está desarrollando y que ha sido cuestionada en diversos foros y congresos educativos nacionales desde las más diversas voces.

Por su parte, la formación docente, tanto la inicial como la llamada continua, ha sido objeto de importantes reformas prácticamente en todo Latinoamérica en las dos últimas décadas (Gatti, 2008). Chile, particularmente, es en ese aspecto, uno de los países de la región, que cuenta con una experiencia más consolidada y documentada desde la instauración entre 1996 y 1997 del Programa de Fortalecimiento de la Formación Inicial Docente. Se crearon ése y otros muchos programas y políticas como la emisión de los Estándares de Desempeño para la Formación Docente. Así mismo, se desarrollaron programas de profesionalización para todos los profesores universitarios dedicados a la formación docente, que salieron al mundo a realizar posgrados.

También se conformaron organismos que velaran por la calidad de los procesos de formación y se ha promovido la discusión nacional sostenida en diferentes ámbitos del país; todo lo cual ha permitido la consolidación de la reforma. Ello ha sido así, pese a los necesarios debates, ajustes y tal vez algunos desencuentros, inevitables, cuando se trata de un campo tan estratégico socialmente.

En general, las reformas educativas se plantean grandes y cíclicos cambios bajo el supuesto de que todo lo anterior debe ser sustituido por diferentes e innovadoras prácticas (Viñao, 2001) y no han sido la excepción aquellas que han pretendido revolucionar a la formación inicial de docentes. Sin embargo, hemos ya comenzado a aprender que no es mediante ese tipo de reformas que conseguiremos los cambios deseados.

Ahora sabemos que los procesos de cambio toman mucho tiempo para introducirse y consolidarse en las realidades educativas, que esos procesos deben promoverse bajo perspectivas integrales y desarrollarse a la par que programas de acompañamiento permanentes y evaluación constante de sus componentes, entre otras cosas.

Cada reforma identifica nuevos "retos" y prioridades, ya sean los de la centuria o aún los del milenio. $Y$ es verdad que en la formación continua de docentes, no sólo en la inicial, nos enfrentamos a retos muy actuales, porque se dan en el presente, pero se asientan sobre otros muy añejos. Son retos que no hemos superado, a pesar de que reformas, acuerdos nacionales, así como reuniones de expertos, foros y congresos nacionales e internacionales van y vienen sin tregua.

Actualmente, con frecuencia planteamos, por ejemplo, que un nuevo reto para los futuros maestros y los que están en servicio, es la incorporación y el uso de las herramientas tecnológicas en la escuela y no podemos negar que se trata de una realidad frente a la cual los maestros deben estar preparados. No obstante, ya escuchamos voces autorizadas y análisis sustentados que nos alertan sobre los riesgos de suponer que estas herramientas representan la solución a nuestros problemas pedagógicos. Nos previenen sobre los riesgos de suponer que la disponibilidad implica acceso y nos recuerdan que los beneficios de toda herramienta dependen del uso social de que sean objeto y que, en el caso de la escuela deberán estar al servicio de la docencia y el aprendizaje, no al revés.

Se dice y tal vez sea posible, que la escuela será otra en la era de la informática, no obstante, literatura reciente nos recuerda que:

La institución escolar vive la paradoja de la época con la instalación de la sala de cómputo mientras el techo se cae y no hay dinero para pagar mejor a los docentes; tensionada entre los múltiples proyectos, programas, propuestas y objetos de diverso tipo que aterrizan en la escuela; atareada ésta tratando de armar el rompecabezas de la última reforma y hacer sentido del conjunto y tan ocupada con todo ello que no le queda tiempo para dedicarse a lo esencial: repensar mientras se hacen, la enseñanza y el aprendizaje (Torres, 2000, p. 10).

Es verdad que la creciente introducción de las nuevas tecnologías a la escuela representa otros retos a la formación inicial de docentes y aún más complejos para los profesores en servicio. Pero también es cierto que no podemos plantearnos la introducción de estos recursos sin vincularla con los retos que aún no superamos en la educación básica. Algunos de ellos son:

La enseñanza efectiva de la alfabetización inicial y las matemáticas

La apropiada atención pedagógica a los alumnos de escuela con grupos multigrado

La atención pedagógica a los alumnos multirepitentes

La evaluación pedagógica de tipo procesual

La interculturalidad en la escuela y el aula

La inclusión educativa en condiciones que realmente la posibiliten en las aulas.

Se trata de cuestiones referidas a la docencia, pero no remediables sólo por ella. Ciertamente deben atenderse desde la formación inicial, pero su solución transita por cambios administrativos, de gestión y de política educativa más generales que promuevan por ejemplo:

El eficaz acompañamiento pedagógico para los docentes en servicio

Aligerar las cargas administrativas y de gestión para los docentes de manera que el tiempo de enseñanza sea mayor y de calidad.

Incorporar el uso de las tecnologías de la información a la formación inicial vinculando su uso a los problemas pedagógicos ya reconocidos y no resueltos.

Introducción a la educación básica de las tecnologías actuales desde las realidades escolares, vinculando su uso a las experiencias docentes de los profesores. 
Promover y posibilitar los tiempos y condiciones institucionales para el trabajo colegiado, entre otros.

\section{Para finalizar}

Finalmente, insistiré en que es necesario reconocer que adscribirnos a los discursos que cíclicamente se presentan como innovadores, no implica mejorar los procesos educativos en nuestras escuelas. Es necesario además, seguir considerando las realidades escolares que no hemos conseguido cambiar y que, por persistentes y reiteradas a muchos pueden parecerles simples o "naturales." Una rápida adscripción a discursos de época que crean nuevas nomenclaturas en educación, como la de competencias (Gatti, 2008), en ocasiones nos impiden reconocer los cambios más profundos que debemos promover en el trabajo escolar y en la formación docente.

Como nos propone Torres:

No se trata de un mero cambio del "rol docente" sino de un cambio profundo del propio modelo escolar; no hay posibilidad de que los docentes asuman un nuevo rol profesional en el marco de un orden escolar atrasado, rígido y jerárquico, pensado para docentes-ejecutores, no para docentes reflexivos, creativos, autónomos (2000, p. 11).

Un nuevo profesionalismo docente no se construye en el vacío, sino apelando a la mejor docencia que nos ha sido legada por generaciones de maestros, así como a las nuevas aportaciones que la ciencia y la tecnología ofrecen para un mejor trabajo escolar. $Y$ con todo ello los nuevos docentes deberán hacer sentido de la paradoja de nuestro tiempo y nuestra región: construir calidad y equidad "desde las limitaciones de la pobreza y las posibilidades de la tecnología, pero también desde las posibilidades de aquella y las limitaciones de ésta" En ese proceso los docentes deben "identificar lo bueno a mantener y lo bueno a incorporar, siempre aprendiendo" (Idem: 27), lo cual es indispensable en cualquier profesión que pretenda contribuir para los cambios que nuestra sociedad demanda.

\section{Referências}

Ávalos, B. (2000). El desarrollo profesional de los docentes. Proyectando desde el presente al futuro. Seminario sobre prospectiva de la educación en la región de América Latina y el Caribe. Santiago de Chile: UNESCO.

Ávalos, B. (2004). La formación docente inicial en Chile. Disponível: www.sica.int/búsqueda/busqueda_archivo.aspx?Archivo=oduc_1 2780_1_01022007.pdf

Clandinin, J., \& Connelly, M. (1996). Teachers' Professional Knowledge Landscapes: Teacher Stories-Stories of Teachers -School Stories -Stories of School. Educational Researchers, 25(3), 24-30.
Cole, M., \& Scribner, S. (1978). Introduction, in Vygotski L. Mind in Society. The Development of Higher Psychological Processes. España: Editorial Crítica.

Encinas, M. A. (2005). Voces magisteriales en torno al Programa Nacional para la Actualización Permanente de los Maestros de Educación Básica en Servicio (PRONAP). Tesis de maestría, Departamento de Investigaciones Educativas del Centro de Investigación y de Estudios Avanzados del Instituto Politécnico Nacional, México.

Espinosa, T. E. (2007). Los maestros y la apropiación de nuevas propuestas pedagógicas. Estudio etnográfico de la incorporación de una reforma para la alfabetización inicial en la primaria. Tesis doctoral en CD, Departamento de Investigaciones Educativas del Centro de Investigación y de Estudios Avanzados del Instituto Politécnico Nacional, México.

Estrada, R. P. (2009). Formación inicial en la normal: Construcción de significados de maestros y estudiantes en las prácticas preservicio. Tesis de doctorado. Departamento de Investigaciones Educativas del Centro de Investigación y de Estudios Avanzados del Instituto Politécnico Nacional, México.

Estrada, R. P., \& Mercado, R. (2008). Procesos de negociación de significado en una escuela normal mexicana. Psicología \& Sociedade, 20(3), 391-401.

Gatti, B. A. (2008) Análise das políticas públicas para formacao continuada no Brasil, na última década. Revista Brasileira de Educacao, 13(37), 57-70.

Hilario, C. N. (2010). Las prácticas escolares de estudiantes de magisterio: entre la normal y la primaria. Tesis de maestría (en proceso), Departamento de Investigaciones Educativas del Centro de Investigación y de Estudios Avanzados del Instituto Politécnico Nacional, México.

Lave, J. (1988). La cognición en la práctica. España: Paidós.

Lave, J., \& Wenger, E. (1991) Situated Learning; Legitimate Peripheral Participation. Cambridge: Cambridge University Press.

Marcelo, G. C. (1999). ¡Aprender a enseñar! ¿Cómo organizar la experiencia práctica en la formación de docentes de manara de fortalecer la calidad de este aprendizaje? Ponencia presentada al panel de fortalecimiento de la formación inicial de docentes. Viña del Mar, Chile.

McLellan, H. (1991) Virtual Environment and Situated Learning. Multimedia Review, 2(3), 30-37.

Mercado, R. (2002). Los saberes docentes como construcción social. La enseñanza centrada en los niños. México: Fondo de Cultura Económica. 
Ministerio de Educación de Chile (MEC). (2000). Estándares de Desempeño para la Formación Inicial de Docentes. Chile.

Rockwell, E., \& Mercado R. (1986). La práctica docente y la formación de maestros. En E. Rockwell \& R. Mercado. La escuela, lugar del trabajo docente. Descripciones y debates (pp. 63-75). México: Cuadernos de Educación DIE, DIE-CINVESTAV-IPN.

Schön, D. A. (1992). La formación de profesionales reflexivos. Barcelona: Paidós-MEC.

Secretaria de Educación Pública. (2003) Hacia una política integral para la formación y el desarrollo profesional de los maestros de educación básica. Cuadernos de discusión 1, Documento base, p. 25

Secretaria de Educación Pública. (2004). Plan de Estudios 2004. Licenciatura en Educación Especial, México.

Schubaver-Leoni, \& Grossen. (1992). The Construction of Adult Child Intersubjetivity in Psychological Research and in School. En M. Von Cronach, W. Doise \& G. Murgny (Eds.), Social Representation and the Social Bases of Knowledge, 1, 69-76.

Souza, D. T. R. de. (2007). O argumento da incompetência e outros discursos na formação de professores. En C. Schwartz, J. M. Carvalho, R. H. Simões \& V. C. Araújo (Org.), Desafios da Educação Básica: a pesquisa em educação (pp. 45-56). Vitória: EDUFES - Editora da Universidade Federal do Espírito Santo.

Tardif, M. (1992). Savoirs et Expérience chez les Enseignants de Métier. Quelques pistes et jalons concernant la nature des savoirs d'expérience. Text présenté lors du Symposium du REF. Sherbrooke, Québec.
Tardif, M., Figueroa, L., Cividini, M., \& Mujawamariya, D. (2000). La formación de maestros en Europa y América del Norte según los nuevos enfoques profesionales de la enseñanza. Propuesta Educativa, 10(22).

Tardif, M., Lessard, C., \& Lahaye, L. (1991). Os Professores face ao saber. Esboço de uma problemática do saber docente. Teoria \& Educaçáo, 4, 215-233.

Torres, R. M. (2000). La profesión Docente en la era de la Informática y la Lucha contra la Pobreza. Reunión del Comité Regional Intergubernamental del Proyecto de Educación, 7. UNESCOOREALC, Santiago.

Vaillant, D. (2002). Formación de formadores: Estado de la Práctica. PREAL: Programa de Promoção da Reforma Educativa na América Latina e Caribe, 25.

Viñao, A. (2001). La cultura de las reformas escolares. Perspectivas docentes, 26, 38-66.

Wenger, E. (1998). Comunidades de práctica. Aprendizaje significativo e identidad. España: Paidós.

Wilson, E. K. (2006) The impact of an alternative model of student teacher supervision: Views of the participants. Teaching and Teacher Education, 22, 22-31.

\section{Sobre a autora}

Ruth Mercado Maldonado (ruthmercadom@gmail.com)

Instituto Politécnico Nacional do México, México D.F.

\section{Correspondência}

Ruth Mercado Maldonado

Calzada de los Tenorios 235, Col. Granjas Coapa, México, D.F., C.P. 14330. 